Original article

\title{
Expression profiles of Annexin A1, formylated peptide receptors and cyclooxigenase-2 in gastroesophageal inflammations and neoplasias
}

\author{
Rodolfo T.C. Takaoka ${ }^{\mathrm{a}}$, Nathália D. Sertório ${ }^{\mathrm{a}}$, Lara P.J. Magalini ${ }^{\mathrm{a}}$, Leandro M. Dos Santos ${ }^{\mathrm{a}}$, \\ Helena R. Souza ${ }^{a}$, Melina M. Iyomasa-Pilon ${ }^{\mathrm{a}}$, Lucas Possebon ${ }^{\mathrm{a}, \mathrm{b}}$, Sara S. Costa ${ }^{\mathrm{a}}$, Ana P. Girol ${ }^{\mathrm{a}, \mathrm{b}, *}$ \\ ${ }^{a}$ Padre Albino Integrated College (FIPA), Department of Physical and Biological Sciences, Catanduva, São Paulo, Brazil \\ ${ }^{\mathrm{b}}$ São Paulo State University, (UNESP), Department of Biology, Laboratory of Immunomorphology, São José do Rio Preto, São Paulo, Brazil
}

\section{A R T I C L E I N F O}

\section{Keywords:}

ANXA1

FPR

Upper digestive tract

Tumors

Inflammation

\begin{abstract}
A B S T R A C T
The anti-inflammatory protein Annexin-A1 (ANXA1) is associated to tumor invasion process and its actions can be mediated by formylated peptides receptors (FPRs). Therefore, we evaluated the expression and correlation of ANXA1, FPR and cyclooxygenase $-2(\mathrm{COX}-2)$ enzyme in esophageal and stomach inflammations and neoplasias. The study of proteins was performed by immunohistochemistry in biopsies of esophagitis, Barrett's esophagus, squamous cell carcinoma and adenocarcinoma of the esophagus, as well as gastritis, stomach polypus and gastric adenocarcinoma. The intensity of the expressions was evaluated by densitometry. The immunohistochemical and densitometric analyzes showed specificity for the FPR1 receptor and modulation of the ANXA1, COX - 2 and FPR1 expressions in the epithelial cells in the different studied conditions. Increased immunoreactivity of these proteins was observed in cases of inflammation and stomach polypus. Interestingly, moderate immunoreactivity for ANXA1 and FPR1 but increased immunolabeling for COX -2 were observed in Barrett́s esophagus and esophageal adenocarcinomas. Also, there was reduced expression of ANXA1 and FPR1 in esophageal carcinoma but COX - 2 overexpression in this tumor. There was no expression of FPR2 but ANXA1 and FPR1 expressions were positively correlated in all clinical conditions studied. Positive correlation between ANXA1 and COX - 2 were also observed in inflammation conditions while negative correlation between ANXA1 and COX -2 was observed in esophageal carcinoma. Our results demonstrate the unregulated expression of ANXA1 and COX -2 in precursor lesions of esophageal and stomach cancers, reinforcing their involvement in gastroesophageal carcinogenesis. In addition, the data show that the actions of ANXA1 in the inflammatory and neoplastic processes of the esophagus and stomach are specifically mediated by the FPR1 receptor.
\end{abstract}

\section{Introduction}

Inflammation and tumors of the esophagus and stomach are important clinical conditions with high incidence and mortality [1-3]. It is known that in many neoplasias, inflammation plays a fundamental role in tumor initiation, progression and metastasis $[2,4,5]$. The pro-inflammatory cyclooxygenase-2 (COX-2) enzyme has been observed to be increased in different tumors and related to the processes of angiogenesis, tumor proliferation and metastasis [5]. Studies have shown the prognostic significance of COX-2 in esophageal adenocarcinomas and carcinomas [6-9], as well as in gastric inflammations and cancers [10-15].
In addition, investigations indicate that COX-2 can be regulated by the antiinflammatory protein annexin A1 (ANXA1) [15,16]. ANXA1 presents tumor-specific expression pattern and is related to the regulation of cell growth, tumor invasion, metastasis, apoptosis and drug resistance, being considered an important target for research related to tumor development [16-19]. But the role of ANXA1 in cancer is conflicting as the protein can be downregulated in some cancers and upregulated in others [19].

The expression of ANXA1 has already been observed in different clinical conditions of the upper digestive system, especially esophageal carcinomas and stomach adenocarcinomas [17-19]. Studies indicate loss of the protein expression in the early stages of tumorigenesis in the

\footnotetext{
Abbreviations: $\mu \mathrm{m}$, Micrometer; Ac2-26, Mimetic peptide Ac2-26 of annexin protein; ADC, Adenocarcinoma; ANOVA, Analysis of variance; ANXA1, Annexin A1Protein; BSA, Albumina

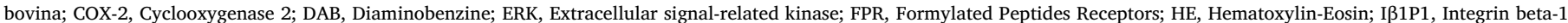

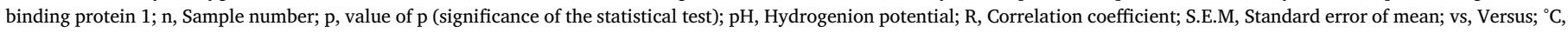
Celsius degree

* Corresponding author. Department of Physical and Biological Sciences, Integrated College Padre Albino Foundation (FIPA), Rua dos Estudantes, 225, Catanduva, SP, Brazil.

E-mail addresses: anapaulagirol@hotmail.com, anapaula.girol@fipa.com.br (A.P. Girol).
} 
esophagus [20-22] and overexpression in gastric carcinogenesis [18,23-26].

Besides, since the biological functions of ANXA1 occur through the interaction of the protein with receptors for formylated peptides (FPRs), the unregulated expression of ANXA1 and FPRs has also been observed in a variety of tumors. [17,19,27-29]. However, reports on the role of ANXA1 in Barrett́s esophagus, precursor metaplasia of esophageal adenocarcinoma are rare [30], and studies on the expression of the protein and its receptors in the stomach polyp are still unknown.

In view of this and the conflicting data found in the literature, in this study we investigated the expression of ANXA1 correlated to the expressions of FPRs and COX-2 in different inflammatory and tumor conditions of the esophagus and stomach.
2. Materials and methods

\subsection{Biopsies}

The analyzes were performed on biopsies of esophagitis, Barrett's esophagus, moderately differentiated squamous cell carcinoma and moderately differentiated esophageal adenocarcinoma, as well as gastritis, stomach polypus and moderately differentiated gastric adenocarcinoma ( $n=20 /$ group). The biopsies were obtained from the files of the Service of Pathology, Faculty of Medicine of the Integrated Colleges Padre Albino of Catanduva, Brazil, after approval of the Committee of Ethics in Research (Protocol 73/11). Fragments of normal esophagus and stomach were used as controls.

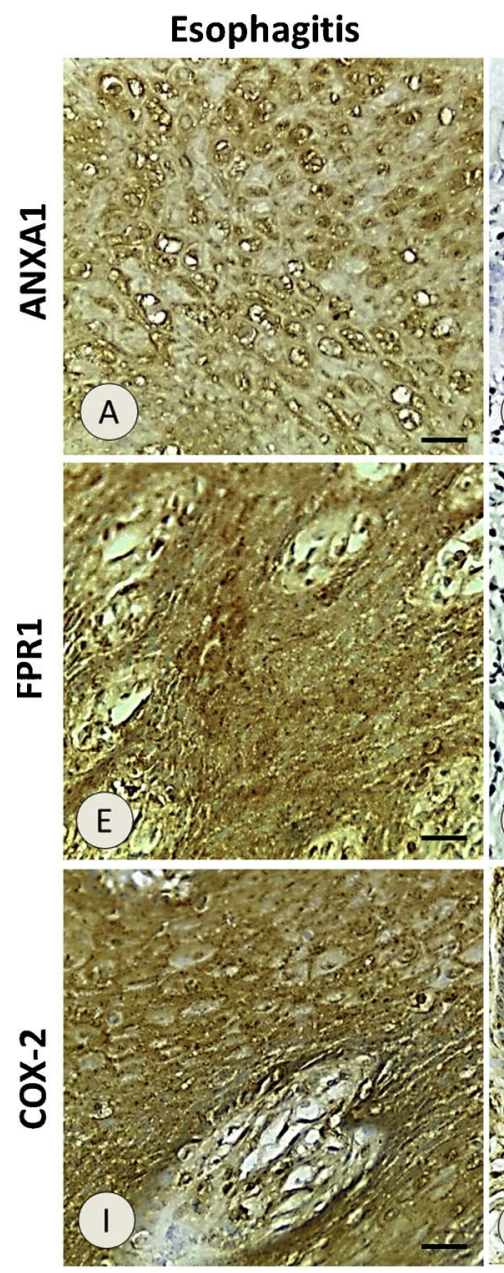

ANXA1

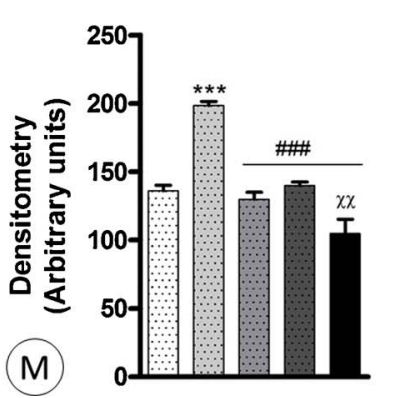

Barret

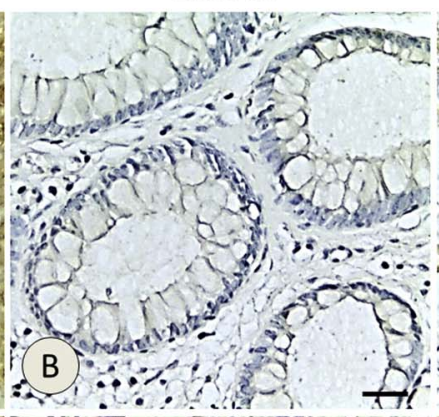

$A D C$

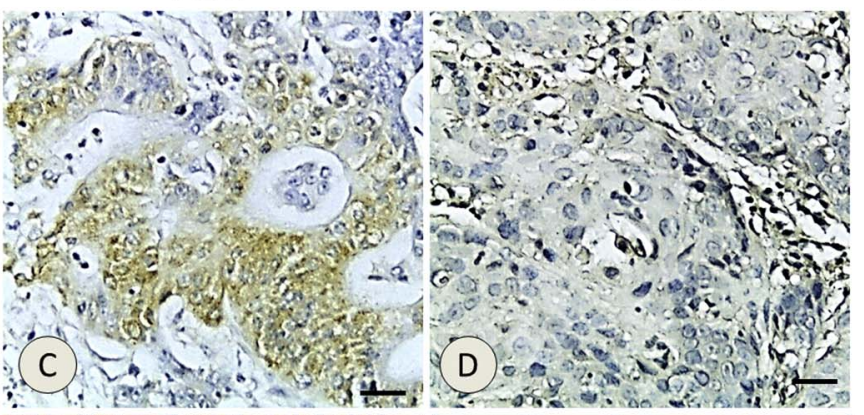

\section{Carcinoma}

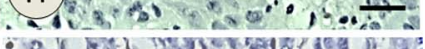
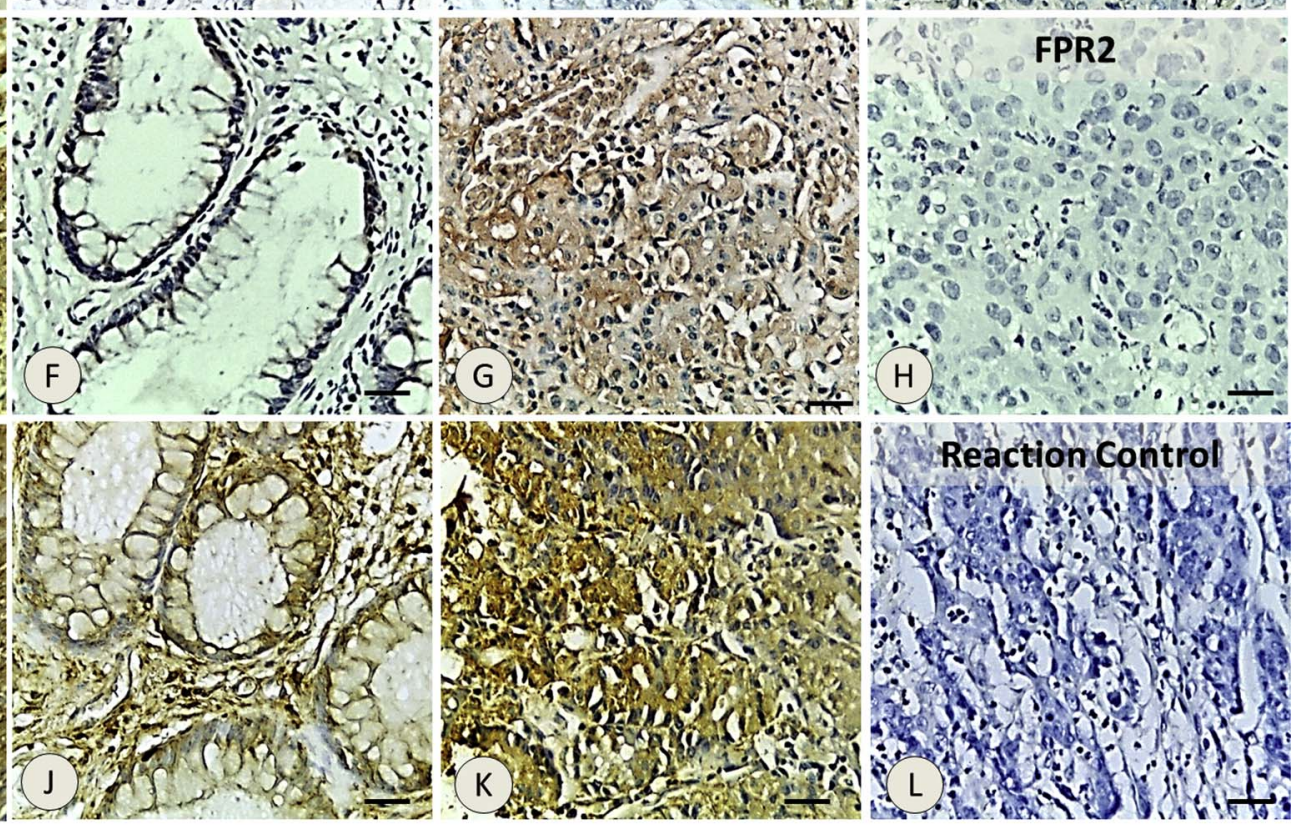

FPR1

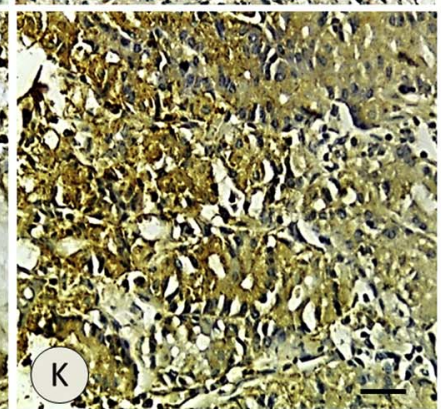

COX-2
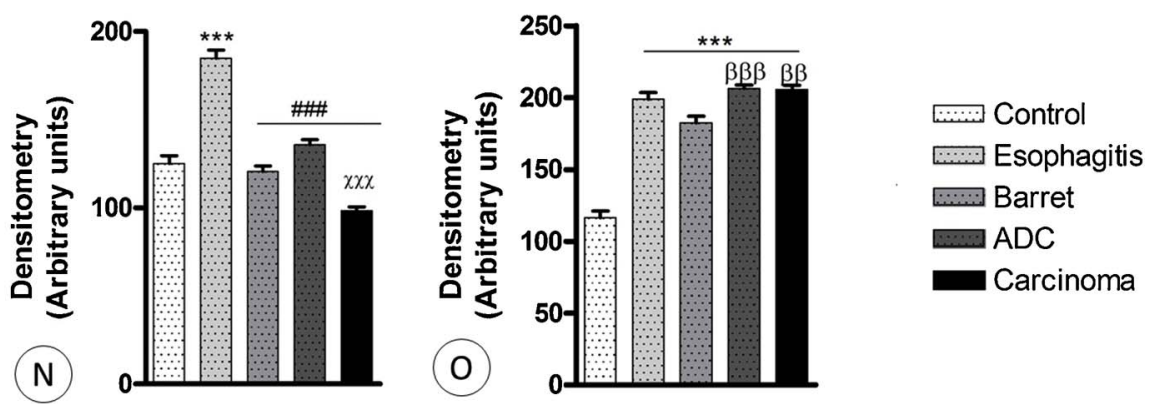

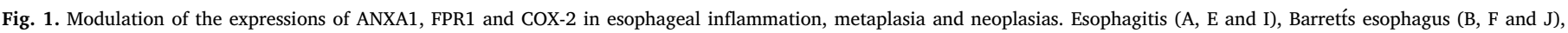

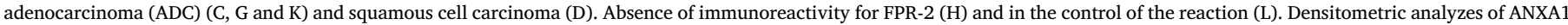

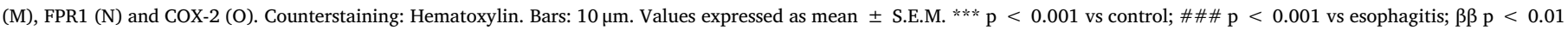
and $\beta \beta \beta \mathrm{p}<0.001$ vs Barretts esophagus; $\chi \chi \mathrm{p}<0.01$ vs control and ADC; $\chi \chi \chi \mathrm{p}<0.001$ vs control, Barrett and ADC. 


\subsection{Histopathological and immunohistochemical studies}

Paraffin included biopsies were used to obtain $5 \mu \mathrm{m}$ sections that were stained by Hematoxylin-Eosin (HE) for histopathological study. Expressions of ANXA1, COX-2, FPR1 and FPR2 proteins were verified in the sections of the selected biopsies, using the following rabbit polyclonal primary antibodies: anti-ANXA1 (1:1000); anti-COX-2 (1:500), anti-FPR1 (1:500) and anti-FPR2 (1:500) (Zymed Laboratories, Cambridge, UK).

The sections were prepared on silanized slides, deparaffinized and rehydrated. After the antigenic recovery (citrate buffer $\mathrm{pH} 6.0$ at $96^{\circ} \mathrm{C}$ for $20 \mathrm{~min}$ ) and endogenous peroxidase blockade they were incubated in a humid chamber at $4{ }^{\circ} \mathrm{C}$ with the primary antibodies diluted in $1 \%$ BSA. Then the sections were incubated with biotinylated secondary antibody (kit Histostain, Invitrogen), developed with DAB substrate (kit $\mathrm{DAB}$, Invitrogen) and counterstained with hematoxylin. The negative control of reaction was performed by omission of the primary antibody.

Analysis of the expression of the proteins in the epithelial cells was performed by optical densitometry (arbitrary units 0-255) using the Leica Image Analysis program. From the images obtained by the $40 \mathrm{x}$ objective in the DM50 microscope (Leica, Alemanha) 20 points were analyzed in 3 different regions to obtain a mean related to the intensity of the immunoreactivity $[25,26]$.

\subsection{Statistical analyzes}

The results were previously submitted to descriptive analysis and determination of normality, and the means were compared by Analysis of Variance (ANOVA), followed by the Bonferroni test. ANXA1 was correlated to the other proteins by the Pearson test. All values were expressed as mean \pm S.E.M., and $P$ values less than 0.05 were considered statistically significant. The correlation results were indicated as positive or negative.

\section{Results}

\subsection{Modulation of ANXA1, FPR1 and COX-2 in esophageal inflammation, metaplasia and tumors}

Immunohistochemical analyzes of the epithelial cells in the different clinical and pathological conditions of the esophagus revealed absence of immunoreactivity for the FPR2 receptor (Fig. 1H) and therefore specific expression for the FPR1 receptor. The specificity of the antibodies was verified by the reaction control (Fig. 1L).

The expressions of the ANXA1, FPR1 and COX-2 proteins were low in the control epithelia and there was overexpression of these proteins in cases of esophagitis (Fig. 1A,E,I,M-O). Significant increases were also observed for COX-2 in the Barrett's esophagus (Fig. 1J), esophageal adenocarcinoma (Fig. 1K) and squamous cell carcinoma with respect to the control epithelium as well in the esophageal tumors compared to Barrett́s esophagus (Fig. 10).

Decreased expressions of ANXA1 and FPR1 were found in the biopsies of Barrets esophagus (Fig. $1 \mathrm{~B}$ and F), esophageal adenocarcinoma (Figure C and G) and squamous cell carcinoma (Fig. 1D) when compared to esophagitis. Besides, the squamous cell carcinoma showed lower expression of ANXA1 in relation to control epithelium and esophageal adenocarcinoma. Reduced expression of FPR1 was also found in squamous cell carcinoma compared to control, Barrett's esophagus and esophageal adenocarcinoma (Fig. $1 \mathrm{M}$ and N). No nuclear labelings of these proteins were verified on tumor cells.

A positive correlation was observed between ANXA1 and FPR1 receptor in all the studied clinics conditions of the esophagus (Table 1). Positive correlation between ANXA1 and COX-2 was also observed in esophagitis. In contrast, ANXA1 and COX-2 showed a negative correlation in esophageal squamous cell carcinoma.
Table 1

Correlation of ANXA1 expression levels between FPR1 and COX-2 in esophageal samples.

\begin{tabular}{lll}
\hline ANXA1 & FPR1 & COX-2 \\
\hline Control & $137,8 \pm 5820$ & $116,5 \pm 4473$ \\
& $\mathrm{R}=0,447$ & $\mathrm{R}=0,10$ \\
& $\mathrm{P}=0,034^{*}$ & $\mathrm{P}=0,363$ \\
Esophagitis & $183,5 \pm 4521$ & $199,1 \pm 4149$ \\
& $\mathrm{R}=0,7169$ & $\mathrm{R}=0,6344$ \\
Barrett & $\mathrm{P}=0,0010^{* *}$ & $\mathrm{P}=0,0033^{* *}$ \\
& $120,5 \pm 3114$ & $182,4 \pm 4700$ \\
& $\mathrm{R}=0,5824$ & $\mathrm{R}=0,0316$ \\
Adenocarcinoma & $\mathrm{P}=0,0102^{*}$ & $\mathrm{P}=0,6231$ \\
& $135,6 \pm 3038$ & $206,4 \pm 2409$ \\
& $\mathrm{R}=0,4225$ & $\mathrm{R}=0,1266$ \\
Carcinoma & $\mathrm{P}=0,0419^{*}$ & $\mathrm{P}=0,3129^{*}$ \\
& $91,46 \pm 2562$ & $205,7 \pm 3081$ \\
& $\mathrm{R}=0,5897$ & $\mathrm{R}=0,6516$ \\
& $\mathrm{P}=0,0095^{* *}$ & $\mathrm{P}=-0,0048^{* *}$ \\
\hline
\end{tabular}

$\mathrm{R}$ = Correlation coefficient; P value: Pearson's correlation; * significant difference

\subsection{Overexpressions of ANXA1, FPR1 and COX-2 in gastric inflammations and neoplasias}

Again there was absence of FPR2 expression (Fig. 2H) and specific immunolabeling of FPR1 in the studied clinic-pathological conditions of the stomach (Fig. 2E-G). The reaction control (Fig. 2L) confirmed the specificity of the reaction. The immunohistochemistry studies of the stomach samples showed overexpression of ANXA1, FPR1 and COX-2 in cases of gastritis (Fig. 2A, E, I, M-O), polypus (Fig. 2B,F,J,M-O) and adenocarcinoma (Fig. 2C, G, K, M-O) compared to normal tissue (Fig. 2D). Increased expression of ANXA1 and FPR1 also occurred in polypus (Fig. 2B and F) and adenocarcinoma (Fig. 2C and G) compared to gastritis (Fig. 2M-O). No nuclear expressions were observed in tumor conditions.

Our studies showed the positive correlation between ANXA1 and FPR1 receptor in gastritis, polypus and stomach adenocarcinoma (Table 2). Positive correlation between ANXA1 and COX-2 was also observed in gastritis and adenocarcinoma of the stomach (Table 2).

\section{Discussion}

The immunohistochemical and densitometrical studies showed overexpression of ANXA1 in esophagitis, moderated expression of the protein in Barrettśs esophagus and esophageal adenocarcinoma and also reduced expressions of ANXA1 in esophageal squamous cell carcinoma. The up-regulation of ANXA1 was also observed in ulcerative colitis patients [31] in the active disease or clinical remission compared to healthy controls and corroborate with our results as well literature findings regarding to the importance of ANXA1 in the resolution of the inflammatory process [32]. Also, the decreased expression of ANXA1 verified in non erosive and especially in erosive reflux disease compared to control esophagic mucosa was associated to the fail of ANXA1 to translocate to cell membrane and consequent impairment of mucosa regeneration [33].

Moreover, an important loss of ANXA1 protein expression was observed in premalignant lesions and squamous carcinomas of esophagus compared with patient-matched normal epithelium [20-22] indicating the essential role of ANXA1 in the maintenance of the normal epithelial phenotype. However, other investigation in esophageal squamous cell carcinoma suggested that nuclear expression of ANXA1 may be potentially used as a prognostic biomarker for this type of tumor [34]. Differently, in our study, we did not find nuclear labeling in esophageal squamous cell carcinoma, which may be due to the fact that the studied biopsies were from more differentiated tumors.

Furthermore, in adenocarcinoma of the esophagus [30] the 


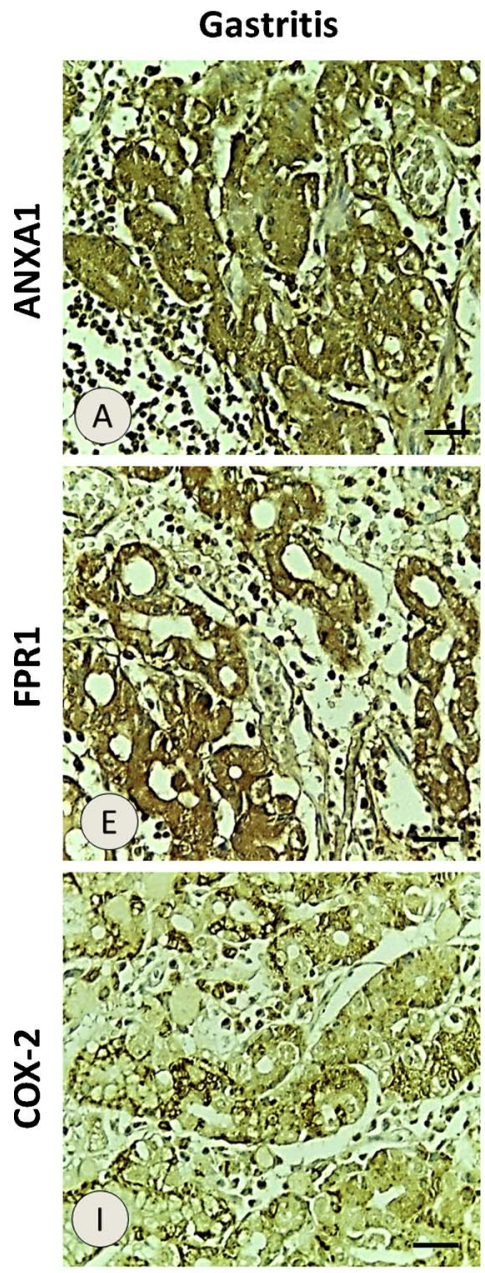

ANXA1

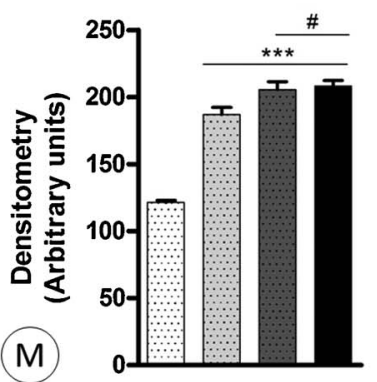

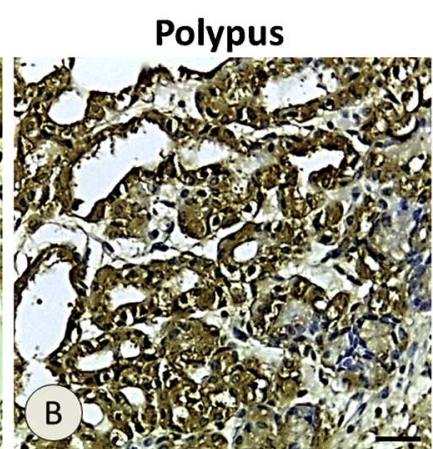

Polypus
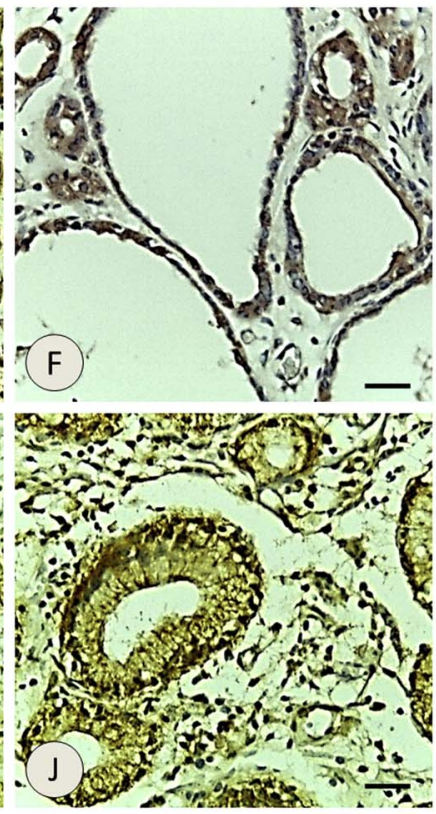

FPR1

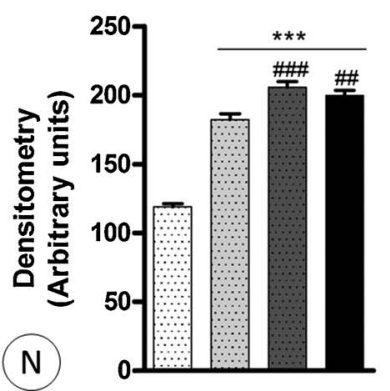

$A D C$
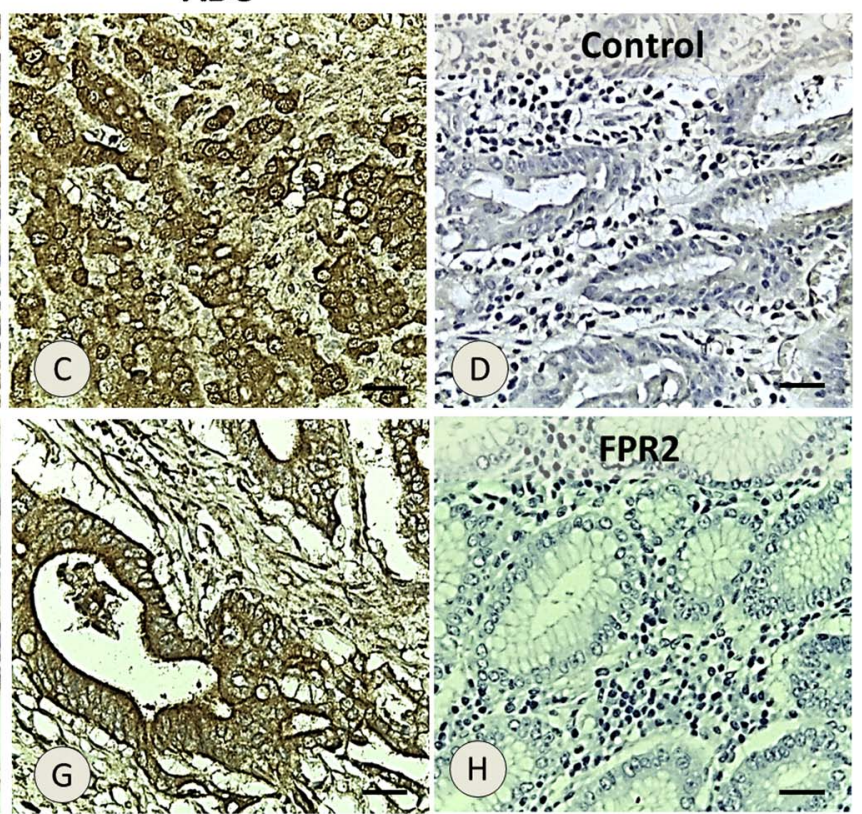
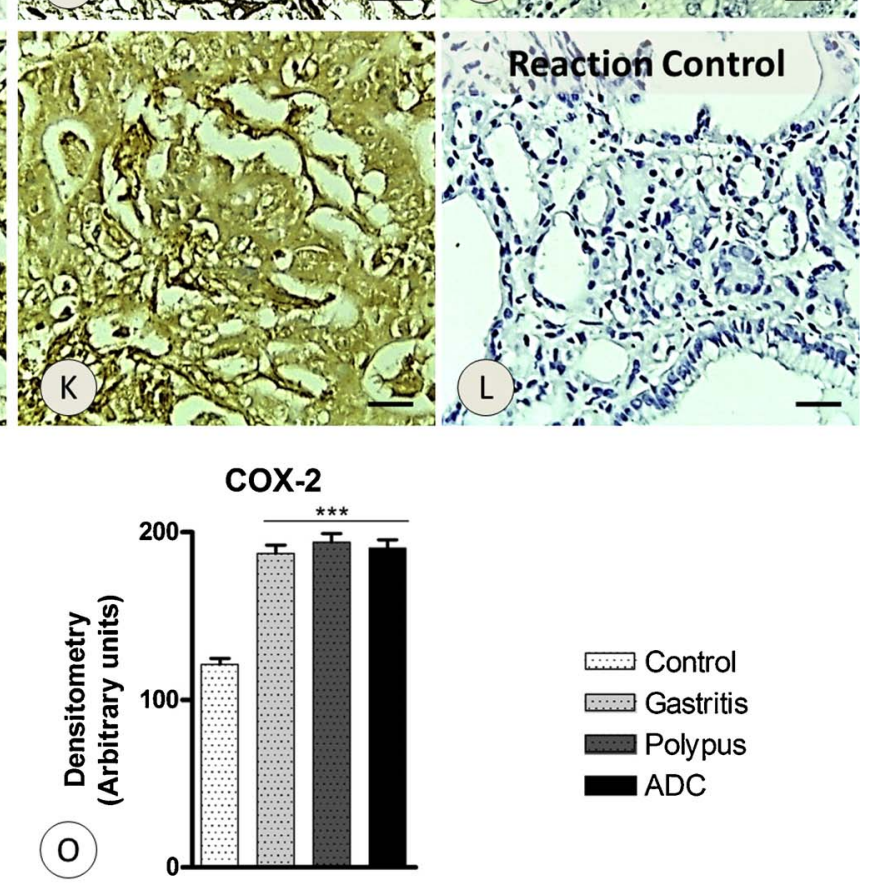

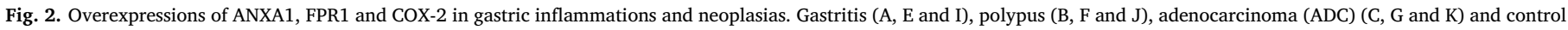

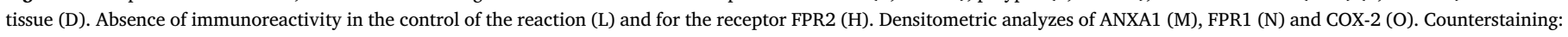
Hematoxylin. Bars: $10 \mu \mathrm{m}$. Values expressed as mean \pm S.E.M. ${ }^{* * *} \mathrm{p}<0.001$ vs normal; \# $\mathrm{p}<0.05$; \#\# $\mathrm{p}<0.01$ and \#\#\# $\mathrm{p}<0.001$ vs gastritis.

upregulation of the microRNA-196a caused the downregulation of ANXA1, with anti-apoptotic effects that allowed adenocarcinoma cell survival. But, the high ANXA1 expression has been demonstrated in esophageal and esophagogastric junction adenocarcinomas and correlated with more advanced pathologic stage and the presence of distant metastasis [35]. Those authors suggested that the role of ANXA1 in esophageal carcinogenesis may be related to the deregulation of arachidonic acid metabolism.

In the continuity of our this research we found higher expression of ANXA1 in inflammatory, benign an malignant conditions of stomach. The up-regulated ANXA1 expression was associated with cancer invasion and lymph node metastasis and high levels of ANXA1 were implicated in poor prognosis of patients [24,36]. The authors pointed that the protein could be considered as a biomarker of clinical prognostic prediction and targeted therapy of gastric adenocarcinoma [36]. ANXA1 was overexpressed in gastritis and gastric cancer [25], and precancerous gastric lesions such as intestinal metaplasia and gastric ulcer [26] suggesting a strong association of the protein with chronic gastric inflammation and carcinogenesis and the ANXA1 involvement in the early stages of gastric carcinogenesis. However, the deregulated expression occurs regardless of the Helicobacter pylori infection and cagA virulence genotype [26].

In an opposite way, the loss of ANXA1 was observed by others researches as gastric cancer progressed and metastasized [37]. Besides, the loss of ANXA1 was associated with the overexpression of COX-2 in clinical gastric cancer, indicating that the anti-proliferative function of ANXA1 against COX-2 production might be lost [15]. The authors found that ANXA1 was widely expressed in normal gastrointestinal epithelium 
Table 2

Correlation of ANXA1 expression levels between FPR1 and COX-2 in gastric samples.

\begin{tabular}{lll}
\hline ANXA1 & FPR1 & COX-2 \\
\hline Control & $118,9 \pm 2302$ & $154,6 \pm 5018$ \\
& $\mathrm{R}=-0,1605$ & $\mathrm{R}=0,0466$ \\
& $\mathrm{P}=02513$ & $\mathrm{P}=0,5490$ \\
Gastritis & $182,2 \pm 4414$ & $187,2 \pm 5037$ \\
& $\mathrm{R}=-0,5087$ & $\mathrm{R}=-0,5425$ \\
& $\mathrm{P}=0,0206^{*}$ & $\mathrm{P}=0,0151^{*}$ \\
Polypus & $205,7 \pm 4185$ & $194,2 \pm 5093$ \\
& $\mathrm{R}=0,4335$ & $\mathrm{R}=-0,0027$ \\
& $\mathrm{P}=0,0038^{*}$ & $\mathrm{P}=0,6495$ \\
Adenocarcinoma & $199,7 \pm 3691$ & $190,6 \pm 4772$ \\
& $\mathrm{R}=0,5066$ & $\mathrm{R}=0,4549$ \\
& $\mathrm{P}=0,0210^{*}$ & $\mathrm{P}=0,0324^{*}$ \\
\hline
\end{tabular}

$\mathrm{R}=$ Correlation coefficient; $\mathrm{P}$ value: Pearson's correlation; *significant difference

and suggested its role in the maintenance of cellular boundaries and regulation of gastric cancer cell viability via the COX-2 pathway [15].

However, our results showed negative ANXA1/COX-2 correlation only in esophageal squamous cell carcinoma, with increased expression of COX-2 e reduced expression of ANXA1, but overexpression of both proteins, which were positively correlated in esophagitis, gastritis, stomach polyp and gastric adenocarcinoma. Although ANXA1 may inhibit COX-2, among other enzymes [38] our data indicated that ANXA1 is unable to control COX-2 expression in the stomach studied conditions.

Regarding COX-2, the increased expressions of the enzyme in inflammatory, benign and malignant conditions of esophagus and stomach studied in this investigation are in line with other researches. Increased COX-2 expression was more characteristic of dysplasia and carcinoma than normal mucosa, suggesting a possible association with cell differentiation and esophageal tumorigenesis [39]. The strong expression of COX-2 was correlated with tumor progression and poor differentiation in esophageal squamous cell carcinoma [9]. In addition, higher expression of COX-2 was associated with carcinogenesis in Barrett's esophagus $[6,40]$ as well as to the significantly reduced survival of patients undergoing surgery for esophageal adenocarcinoma $[7,8]$.

COX-2 expression was suggested as an indicator for intestinal type carcinoma, advanced gastric tumor and H. pylori infection [12]. Also COX-2 mRNA expression in gastric carcinoma tissue was correlated with depth of invasion, indicating that this enzyme is involved in the growth of stomach tumor [10]. Other investigation indicated that COX2 expression induced by $\mathrm{H}$ pylori infection is an early event during gastric carcinogenesis [11]. Besides, it was suggested that COX-2 overexpression can be associated with increased prostaglandin- $\mathrm{E}_{2}$ biosynthesis and angiogenesis in gastric cancer [14], once again indicating this enzyme involvement in the development of stomach cancer.

After observing the ANXA1 expression we analyzed the FPRs profiles in the upper digestive tract biopsies. Our data showed modulation of FPR1 in esophageal biopsies similar to the ANXA1 expression. High expression of FPR1 overlapping the ANXA1 immunolocalization was also found in gastritis, polypus and adenocarcinoma of stomach. The FPR2 expression was not observed in none of the studied conditions of esophagus and stomach, indicating the ANXA1/FPR1 specific interaction.

The association ANXA1/FPR was also observed in other investigations [23,28,29]. The expression of ANXA1 was increased in the gastric ulcer margin throughout the healing process. Also, the treatment with an ANXA1 mimetic (Ac2-26) significantly enhanced gastric ulcer healing while the FPR antagonist impaired the early phase of the healing process [23]. ANXA1 expression was positively correlated with invasiveness of human gastric cancer cells both in vitro and in vivo [28], but the authors pointed that ANXA1 could trigger the three FPRs
(FPR1, FPR2 and FPR3) and stimulate cell invasion by extracellular signal-related kinase (ERK) phosphorylation and subsequent integrin $\beta 1$-binding protein 1 (I 1 1P1) expression. Similar to our data other investigation reported that FPR1 expression increased significantly when ANXA1 overexpression was present in gastric cancer cells [29], indicating that a positive feedback regulation of FPR1 is involved in the ANXA1-FPR1 signal transduction.

\section{Conclusion}

Our data show that the actions of ANXA1 in the inflammatory and neoplastic processes of the esophagus and stomach are specifically mediated by the FPR1 receptor and indicate the deregulation of ANXA1/FPR1 as well the COX-2 overexpression in inflammation, metaplasia and benign and malignant neoplasias of the esophagus and stomach, reinforcing the involvement of these proteins in gastro-esophageal carcinogenesis.

\section{Disclosure}

All authors declare that are no conflicts of interest.

\section{References}

[1] R. Siegel, C. DeSantis, K. Virgo, K. Stein, A. Mariotto, T. Smith, D. Cooper, T. Gansler, C. Lerro, S. Fedewa, C. Lin, C. Leach, R.S. Cannady, H. Cho, S. Scoppa, M. Hachey, R. Kirch, A. Jemal, E. Ward, Cancer treatment and survivorship statistic, 2012, CA. Cancer J. Clin. 62 (4) (2012) 220-241.

[2] A. Jemal, F. Bray, M.M. Center, J. Ferlay, E. Ward, D. Forman, Global cancer statistic, CA. Cancer J. Clin. 61 (2) (2011) 69-90.

[3] S. Torres, E.E. de la Riva, L.S. Tom, M.L. Clayman, C. Taylor, X. Dong, M.A. Simon, The development of a communication tool to facilitate the cancer trial recruitment process and increase research literacy among underrepresented populations, J. Cancer Educ. 30 (4) (2015) 792-798.

[4] D.G. DeNardo, M. Johansson, L.M. Coussens, Inflaming gastrointestinal oncogenic programming, Cancer Cell. 14 (1) (2008) 7-9.

[5] J. Todoric, L. Antonucci, M. Karin, Targeting inflammation in cancer prevention and terapy, Cacer Prev. Res. (Phila) 9 (12) (2016) 895-905.

[6] V.N. Shirvani, R. Ouatu-Lascar, B.S. Kaur, G. Triadafilopoulus, Cyclooxygenase 2 expression in Barrett's esophagus and adenocarcinoma: ex vivo induction by bile salts and acid exposure, Gastroenterology 118 (3) (2000) 487-496.

[7] C.J. Buskens, A. Van Rees, C. Reitsma, P.J. Bosma, G.J. Offerhaus, J.J. Van Lanchott, A. Ristimäki, Prognostic significance of elevated cyclooxygenase 2 expression in patients with adenocarcinoma of the esophagus, Gastroenterology 122 (7) (2002) 1800-1807.

[8] M. France, P.A. Drew, T. Dodd, D.I. Watson, Cyclo-oxygenase-2 expression in esophageal adenocarcinoma as a determinant of clinical outcome following esophagectomy, Dis. Esophagus 17 (2) (2004) 136-140.

[9] N. Hashimoto, M. Inayama, M. Fujishima, H. Shiozaki, Clinicopathologic significance of expression of cyclooxygenase- 2 in human esophageal squamous cell carcinoma, Hepatogastroenterology 54 (75) (2007) 758-760.

[10] K. Ohno, T. Yoshinaga, K. Fujita, H. Hasegawa, H. Iseki, W. Tsunozaki, Z. Ichikawa, Depth of invasion parallels increased cyclooxygenase-2 levels in patients with gastric carcinoma, Cancer 91 (10) (2001) 1876-1881.

[11] W.H. Sun, Q. Yu, H. Shen, X.L. Ou, D.Z. Cao, T. Yu, C. Qian, F. Zhu, Y.L. Sun, X.L. Fu, H. Su, Roles of Helicobacter pylori infection and cyclooxygenase-2 expression in gastric carcinogenesis, World J. Gastroenterol. 10 (19) (2004) 2809-2813.

[12] N.H. Hafez, N.S. Tahoun, Expression of cyclooxygenase 2 and vascular endothelial growth factor in gastric carcinoma: relationship with clinicopathological parameters, J. Egypt Natl. Canc. Inst. 28 (3) (2016) 149-156.

[13] X.Y. Zhang, P.Y. Zhang, M.A. Aboul-Soud, From inflammation to gastric cancer: role of Helicobacter pylori, Oncol Lett. 13 (2) (2017) 543-548.

[14] K. Uefuji, T. Ichikura, H. Mochizuki, Cyclooxygenase-2 expression is related to prostaglandin biosynthesis and angiogenesis in human gastric cancer, Clin. Cancer Res. 6 (1) (2000) 135-138.

[15] Y. Gao, Y. Chen, D. Xu, J. Wang, G. Yu, Differential expression of ANXA1 in benign human gastrointestinal tissues and cancers, BMC Cancer 14 (2014) 520.

[16] L. Lim, S. Pervaiz, Annexin 1: the new face of an old molecule, FASEB J. 21 (4) (2007) 968-975.

[17] C. Guo, S. Liu, M.Z. Sun, Potential role of Anxa1 in cancer, Future Oncol. 9 (11) (2013) 1773-1793.

[18] M. Kanda, Y. Kodera, Molecular mechanisms of peritoneal dissemination in gastric cancer, World J. Gastroenterol. 22 (30) (2016) 6829-6840.

[19] B. Boudharaa, C. Bouchon, M. D'Incan, F. Degoul, Annexin A1 localization and its relevance to cancer, Clin. Sci. (Lond.) 130 (4) (2016) 205-220.

[20] C. Pawaletz, D.K. Ornstein, M.J. Roth, V.E. Bichsel, J.W. Gillespie, V.S. Calvert, C.D. Vocke, S.M. Hewitt, J. Duray, N. Wang, W.N. Linehan, P.R. Taylor, L.A. Liotta, 
M.R. Emmert-Buck, E.F. 3rd Petricoin, Loss of annexin 1 correlates with early onset of tumorigenesis in esophageal and prostate carcinoma, Cancer Res. 60 (22) (2000) 6293-6297.

[21] S.H. Xia, L.P. Hu, H. Hu, W.T. Ying, X. Xu, Y. Cai, Y.L. Han, B.S. Chen, F. Wei, X.H. Qian, Y.Y. Cai, Y. Shen, M. Wu, M.R. Wang, Three isoforms of annexin I are preferentially expressed in normal esophageal epithelia but down-regulated in esophageal squamous cell carcinomas, Oncogene 21 (43) (2002) 6641-6648.

[22] N. Hu, M.J. Flaig, H. Su, J.Z. Shou, M.J. Roth, W.J. Li, C. Wang, A.M. Goldstein, G. Li, M.R. Emmert-Buck, P.R. Taylor, Comprehensive characterization of annexin I alterations in esophageal squamous cell carcinoma, Clin. Cancer Res. 10 (18 Pt 1) (2004) 6013-6022.

[23] G. Martin, M. Perreti, R.J. Flower, J.L. Wallace, Annexin-1 modulates repair of gastric mucosal injury, Am. J. Physiol. Gastrointest. Liver Physiol. 294 (3) (2008) G764-9.

[24] Y. Sato, K. Kumamoto, K. Saito, H. Okayama, S. Hayase, Y. Kofunato, K. Miyamoto, I. Nakamura, S. Ohki, Y. Koyama, S. Takenoshita, Up-regulated Annexin A1 expression in gastrointestinal cancer is associated with cancer invasion and lymph node metastasis, Exp. Ther. Med. 2 (2) (2011) 239-243.

[25] Y.C. Jorge, M.M. Mataruco, L.P. Araújo, A.F. Rossi, J.G. de Oliveira, M.C. Valsechi, A. Caetano, K. Miyazaki, C.S. Fazzio, J.A. Thomé, P. Rahal, S.M. Oliani, A.E. Silva, Expression of annexin-A1 and galectin-1 anti-inflammatory proteins and mRNA in chronic gastritis and gastric cancer, Mediators Inflamm. 2013 (2013) 152860.

[26] A.F. Rossi, M.C. Duarte, A.B. Poltronieri, M.C. Valsechi, Y.C. Jorge, D. de-Sant Neto, P. Rahal, S.M. Oliani, A.E. Silva, Deregulation of annexin-A1 and galectin-1 expression in precancerous gastric lesions: intestinal metaplasia and gastric ulcer, Mediators Inflamm. 2014 (2014) 478138.

[27] S. Mussunoor, G.I. Murray, The role of annexins in tumour development and progression, J. Pathol. 216 (2) (2008) 131-140.

[28] T.Y. Cheng, M.S. Wu, J.T. Lin, M.T. Lin, C.T. Shun, H.Y. Huang, K.T. Hua, M.L. Kuo, Annexin A1 is associated with gastric cancer survival and promotes gastric cancer cell invasiveness through the formyl peptide receptor/extracellular signal-regulated kinase/integrin beta-1-binding protein 1 pathway, Cancer 118 (23) (2012) 5757-5767.

[29] T.Y. Cheng, M.S. Wu, J.T. Lin, M.T. Lin, C.T. Shun, K.T. Hua, M.L. Kuo, Formyl Peptide receptor 1 expression is associated with tumor progression and survival in gastric cancer, Anticancer Res. 34 (5) (2014) 2223-2229.

[30] R. Luthra, R.R. Singh, M.G. Luthra, Y.X. Li, C. Hannah, A.M. Romans, B.A. Barkon, S.S. Chen, J. Ensor, D.M. Maru, R.R. Broaddus, A. Rashid, C.T. Albarracin,
MicroRNA-196a targets annexin A1: a microRNA-mediated mechanism of annexin A1 downregulation in cancers, Oncogene 27 (52) (2008) 6667-6678.

[31] L. Vong, J.G. Ferraz, N. Dufton, R. Panaccione, P.L. Beck, P.M. Sherman, M. Perreti, J.L. Wallace, Up-regulation of Annexin-A1 and lipoxin A(4) in individuals with ulcerative colitis may promote mucosal homeostasis, PLoS One 7 (6) (2012) e39244.

[32] L.O. Perucci, M.A. Sugimoto, K.B. Gomes, L.M. Dusse, M.M. Teixeira, L.P. Souza Annexin, A1 and specialized proresolving lipid mediators: promoting resolution as a therapeutic strategy in human inflammatory diseases, Expert Opin. Ther. Targets 21 (9) (2017) 879-896.

[33] C. Calabrese, V. Marzano, A. Urbani, G. Lazzarini, M.C. Valerii, G. Liguori, S. Di Molfetta, F. Rizzello, P. Gionchetti, M. Campieri, E. Spisni, Distinct proteomic profiles characterise non-erosive from erosive reflux disease, Aliment. Pharmacol. Ther. 34 (8) (2011) 982-993.

[34] G. Hang, H. Duan, H. Sheng, J. Huang, Association of nuclear annexin A1 with prognosis of patients with esophageal squamous cell carcinoma, Int. J. Clin. Exp. Pathol. 7 (2) (2014) 751-759.

[35] K. Wang, T.T. Wu, E. Resetkova, H. Wang, A.M. Correa, W.L. Hofstetter, S.G. Swisher, J.A. Ajani, A. Rashid, S.R. Hamilton, C.T. Albarracin, Expression of annexin A1 in esophageal and esophagogastric junction adenocarcinomas: association with poor outcome, Clin. Cancer Res. 12 (15) (2006) 4598-4604.

[36] Z.Q. Zhang, X.J. Li, G.T. Liu, Y. Xia, X.Y. Zhang, H. Wen, Identification of Annexin A1 protein expression in human gastric adenocarcinoma using proteomics and tissue microarray, World J. Gastroenterol. 19 (43) (2013) 7795-7803.

[37] G. Yu, J. Wang, Y. Chen, X. Wang, J. Pan, Q. Li, K. Xie, Tissue microarray analysis reveals strong clinical evidence for a close association between loss of annexin A1 expression and nodal metastasis in gastric cancer, Clin. Exp. Metastasis 25 (7) (2008) 695-702.

[38] M.A. Lizarbe, J.I. Barrasa, N. Olmo, F. Gavilanes, J. Turnay, Annexin-phospholipid interactions. functional implications, Int. J. Mol. Sci. 14 (2) (2013) 2652-2683.

[39] M. Kase, S. Osaki, K. Honjo, H. Hashimoto, S. Adachi, Expression of cyclooxygenase- 1 and cyclooxygenase- 2 in human esophageal mucosa, dysplasia and carcinoma, Pathobiology 71 (2) (2004) 84-92.

[40] N.S. Buttar, K.K. Wang, O. Leontovich, J.Y. Westcott, R.J. Pacifico, M.A. Anderson, K.K. Krishnadath, L.S. Lutzke, L.J. Burgart, Chemoprevention of esophageal adenocarcinoma by COX-2 inhibitors in an animal model of Barrett's esophagus, Gastroenterology 122 (4) (2002) 1101-1112. 